\title{
Erratum: Spin Ferroquadrupolar Order in the Nematic Phase of FeSe [Phys. Rev. Lett. 116, 247203 (2016)]
}

\author{
Zhentao Wang, Wen-Jun Hu, and Andriy H. Nevidomskyy \\ (Received 7 February 2017; published 27 February 2017)
}

DOI: 10.1103/PhysRevLett.118.099901

While discussing the spectrum of spin fluctuations in Fig. 3, it was remarked that the Goldstone modes associated with an alternative antiferroquadrupolar (AFQ) scenario [1] would contradict the inelastic neutron scattering (INS) data on FeSe. We would like to clarify that, given the instrumental and damping broadening in both momentum and energy of the existing INS data [2], it is equally possible to interpret them as being consistent with the AFQ scenario, as stressed by Lai et al. [3]. Specifically, the $\boldsymbol{q}$-integrated low-energy spectral weight near the wave vector $(\pi, 0)$ [the so-called stripe component in Fig. 4(b) of Ref. [2]] appears to be linear in energy from the threshold energy just above the range under the influence of superconductivity (about $10 \mathrm{meV}$ ) up to about $40 \mathrm{meV}$. Such a linear dependence is consistent with the expected behavior in the AFQ phase because this phase preserves the time-reversal symmetry and the low-energy spin spectral weight of the quadrupolar Goldstone mode scales linearly with energy. Such a linear dependence is also expected in the FQ scenario when the spectral weight is integrated over momentum near the wave vector $(0,0)$, which remains to be tested experimentally.

[1] R. Yu and Q. Si, Phys. Rev. Lett. 115, 116401 (2015).

[2] Q. Wang, Y. Shen, B. Pan, X. Zhang, K. Ikeuchi, K. Iida, A. D. Christianson, H. C. Walker, D. T. Adroja, M. Abdel-Hafiez, X. Chen, D. A. Chareev, A. N. Vasiliev, and J. Zhao, Nat. Commun. 7, 12182 (2016).

[3] H.-H. Lai, W.-J. Hu, R. Yu, and Q. Si, arXiv:1603.03027. 